\title{
Single-atom sites on perovskite chips for record-high sensitivity and quantification in SERS
}

\author{
Ran Feng ${ }^{1 \dagger}$, Qing $\mathrm{Miao}^{2 \dagger}$, Xiang Zhang ${ }^{3 \dagger}$, Peixin Cui ${ }^{4 \dagger}$, Cong Wang ${ }^{1 *}$, Yibo Feng ${ }^{1}$, Liyong Gan ${ }^{3}$, \\ Jiaxing $\mathrm{Fu}^{6}$, Shibo Wang ${ }^{7}$, Ziyi $\mathrm{Dai}^{8}$, Liming $\mathrm{Hu}^{5}$, Yunjing Luo ${ }^{5}$, Weihai Sun ${ }^{7}$ Xiaoxian Zhang ${ }^{2}$, \\ Jiawen $\mathrm{Xiao}^{1}$, Jinbo $\mathrm{Wu}^{6}$, Bingpu Zhou ${ }^{8}$, Mingqiang Zou ${ }^{9}$, Dawei $\mathrm{He}^{2}$, Xiaoyuan Zhou ${ }^{3}$ and \\ Xiaodong $\operatorname{Han}^{1 *}$
}

\begin{abstract}
Surface enhanced Raman scattering (SERS) is a rapid and nondestructive technique that is capable of detecting and identifying chemical or biological compounds. Sensitive SERS quantification is vital for practical applications, particularly for portable detection of biomolecules such as amino acids and nucleotides. However, few approaches can achieve sensitive and quantitative Raman detection of these most fundamental components in biology. Herein, a noblemetal-free single-atom site on a chip strategy was applied to modify single tungsten atom oxide on a lead halide perovskite, which provides sensitive SERS quantification for various analytes, including rhodamine, tyrosine and cytosine. The single-atom site on a chip can enable quantitative linear SERS responses of rhodamine $\left(10^{-6}-1 \mathrm{mmol} \mathrm{L}^{-1}\right)$, tyrosine $(0.06-$ $1 \mathrm{mmol} \mathrm{L}^{-1}$ ) and cytosine $\left(0.2-45 \mathrm{mmol} \mathrm{L}^{-1}\right)$, respectively, which all achieve record-high enhancement factors among plasmonic-free semiconductors. The experimental test and theoretical simulation both reveal that the enhanced mechanism can be ascribed to the controllable single-atom site, which can not only trap photoinduced electrons from the perovskite substrate but also enhance the highly efficient and quantitative charge transfer to analytes. Furthermore, the label-free strategy of single-atom sites on a chip can be applied in a portable Raman platform to obtain a sensitivity similar to that on a benchtop instrument, which can be readily extended to various biomolecules for low-cost, widely demanded and more precise point-of-care testing or in-vitro detection.
\end{abstract}

Keywords: SERS, single-atom site, point-of-care testing, in-vitro diagnosis, charge-transfer mechanism, lead halide perovskite

\section{INTRODUCTION}

Surface enhanced Raman scattering (SERS) has been intensively studied for fingerprint tracing of biological and chemical molecules [1], which has a wide variety of applications, such as in food safety, chemical reaction monitoring and medical diagnostics [2-4]. Regarding the widely-applied and robust techniques of electrophoresis, fluorescence and chromatography, they usually exhibit ultrasensitive capabilities and quantitative linear relationships over a large range from the nanomole (single-molecule level) to mole level, which accelerate their applications in various biochemical analyses, including cell, protein and nucleic acid analyses [5-7]. However, as a powerful analytical technique, the major weakness of applying SERS in biological applications is the low sensitivity to nucleotides and amino acids and lack of quantification of Raman scattering signals [3,5]. As the primary and fundamental elements, nucleotides and amino acids are important biomolecules among living organisms in most life processes. Amino acids are building blocks of proteins that can yield various information, while nucleotide detection can provide gene information. Although several analytical methods have quantitative analysis capability, the next technological revolution of in-vitro diagnosis (IVD) and point-of-care testing (POCT) is considered to be fast, direct and highly sensitive analysis of nucleotides and amino acids with precise structural features [8]. Thus, for SERS, sensitive and quantitative analysis of amino acids or nucleotide molecules is a challenging task but of enormous value. Moreover, in the recent years, the attractiveness of portable Raman instruments for POCT/IVD detection has been significantly growing due to their portability and economic advantages [9]. These advantages can

\footnotetext{
${ }^{1}$ Beijing Key Laboratory of Microstructure and Properties of Solids, Institute of Microstructure and Property of Advanced Materials, Faculty of Materials and Manufacturing, Beijing University of Technology, Beijing 100124, China

${ }^{2}$ Key Laboratory of Luminescence and Optical Information, Ministry of Education, Institute of Optoelectronic Technology, Beijing Jiaotong University, Beijing 100044, China

${ }^{3}$ College of Physics and Center for Quantum Materials and Devices, Analytical and Testing Center, Chongqing University, Chongqing 401331, China

${ }^{4}$ Key Laboratory of Soil Environment and Pollution Remediation, Institute of Soil Science, Chinese Academy of Sciences, Nanjing 210008, China

${ }^{5}$ Faculty of Environment and Life, Beijing Key Laboratory of Environmental and Oncology, Beijing University of Technology, Beijing 100124, China

${ }^{6}$ Materials Genome Institute, Shanghai University, Shanghai 200444, China

${ }^{7}$ College of Materials science and Engineering, Huaqiao University, Xiamen 361021, China

${ }^{8}$ Joint Key Laboratory of the Ministry of Education, Institute of Applied Physics and Materials Engineering, University of Macau, Avenida da Universidade, Taipa, Macau 999078, China

${ }^{9}$ Chinese Academy of Inspection and Quarantine (CAIQ), Beijing 100123, China

† The authors contributed equally to this work.

* Corresponding authors (emails: smartswang@bjut.edu.cn (Wang C); xdhan@bjut.edu.cn (Han X))
} 
dramatically expand the application of SERS to diagnosis (such as COVID-19), emergency supervision and homeland security with broad prospects. However, compared with those in conventional Raman confocal setups, the unfocused laser spots and low-end charge-coupled devices may decrease the sensitivity to analytes, especially for complex biomolecules, which becomes a bottleneck for widespread portable Raman application. A highly sensitive, uniform and robust SERS substrate should overcome the weakness of portable Raman spectroscopy and solve this problem together.

To achieve the sensitive and quantitative analysis of biological molecules, the SERS substrate has been extensively developed via two main strategies, i.e., high enhancement factor (EF) for high sensitivity and controllable sites for quantification. For high sensitivity, Huang et al. [10] developed an electro-plasmonic method to identify a single amino acid among 20 distinct multiplexed amino acid signals on Au nanostars. Kim et al. [11] successfully designed mildly selective self-assembled monolayers to capture various DNA or proteins in complex cell lysates on $\mathrm{Au}$ substrates. However, since the precise control of electric fields at hotspots is essentially difficult, especially at the atomic scale, the quantitative SERS based on the electric field faces a severe bottleneck for noble-metal-based detection. Although several quantitative analyses have been realized by the accurate label/tag/condensed techniques on noble metals [12-16], the randomly distributed hotspots lead to considerable uncertainty, resulting in poor reproducibility of the quantitative SERS signals on metallic substrates $[17,18]$. Thus, the discovery of plasmonfree SERS platform via low-cost and scalable approaches is of great interest. In recent years, semiconductor chips/materials based on another mechanism, the chemical enhancement mechanism (CM), have attracted more attention for SERS. Due to the quantitative formation of charge transfer between the sites and nearby molecules, quantitative analysis can be mostly achieved by semiconductor substrates, such as metallic $\mathrm{W}_{18} \mathrm{O}_{49}$, $\mathrm{Ta}_{2} \mathrm{O}_{5}, \delta$-MoN nanosheets, atomic vacancies/two-dimensional (2D) $-\mathrm{TiO}_{2}, \mathrm{ZnO}, \mathrm{Ti}_{3} \mathrm{C}_{2}$, conductive polymers [19-25], 2D materials [26,27] and metal-organic frameworks (MOFs) $[28,29]$. However, novel semiconductors need to be further explored for superior sensitivity. Enhancement of charge transfer (quantum yield) on semiconductors for single-molecule level SERS has drawn considerable attention via further (1) improvement of the photoinduced charge generation [30] and (2) strengthening of the interaction between sites and probe biomolecules [17,31-33]. Furthermore, along these two lines, the understanding of SERS enhancement mechanism could be greatly deepened and enriched. For instance, as important charge-transfer parameters, the quantum yield and lifetime are extremely difficult to quantify and are rarely reported. Thus, an in-depth understanding of the charge transfer is required in semiconductor SERS, and a universal strategy of semiconductor substrate development based on charge transfer is urgently in demand.

Recently, along these two lines, Sun et al. [34] found that SERS signals can partially originate from the modulated photoluminescence $(\mathrm{PL})$ on noble metal, which is different from traditional cognition that the PL usually causes the undesirable background of noise in SERS detection due to photon scattering mechanisms [5]. Interestingly, Yang et al. [35] studied the charge-transfer mechanisms on $\mathrm{Nd}$-doped $\mathrm{ZnO}$ to further reveal that PL quenching is correlated with the SERS improvement rather than Raman scattering noise. Hence, new guidelines for photoinduced generation of SERS signals from semiconductor substrates can be further developed by manipulating chargetransfer sites. Moreover, due to that various amino acids or DNA fragments have diverse energy levels, the optimized semiconductor energy level can potentially provide a suitable band structure for the charge transfer. Coherently, an inorganicbased ternary heterostructure $\mathrm{Fe}_{3} \mathrm{O}_{4} @ \mathrm{GO} @ \mathrm{TiO}_{2}$ SERS substrate was synthesized to obtain remarkable charge-transfer sites via a precise charge-transfer path formed by a tandem nanostructure [36]. Besides, an organic semiconductor site for charge transfer has also been proposed and extensively studied [25], leading to a large SERS EF via a charge-transfer-favorable path and effective molecular interactions. Thus, this strategy of tandem chargetransfer or novel charge-transfer sites can potentially be applied in the design of SERS substrates via nanostructure manipulation to produce a quantitative and ultrasensitive SERS detection.

Since charge transfer and interactions occur on the sites at the several-nanometers level, an in-depth understanding of the energy level and quantum yield can be more extensively investigated at the atomic scale, which potentially provides a broad playground for research in this community $[1,5]$. At the atomic scale, sites have recently been intensely investigated to understand the relationship between sites and target molecules [37]. Downsizing sites to the smallest limit is an extensively applied strategy, including single-atom sites, single-atom oxides or ultrathin-layer materials [38-40]. The precise sites and their charge-transfer mechanism should be increasingly investigated to determine the charge-transfer behavior in terms of thermodynamics and kinetics: photoinduced electron generation, charge-transfer paths and quantum efficiency. Herein, we developed controllable single-atom oxide sites on semiconductor chips, i.e., monodispersed sites of single tungsten atom oxide on a lead halide perovskite. The advantage of the design is that the perovskite is considered as an excellent PL substrate providing sufficient photoinduced charge [41], and a large number of monodispersed sites on the planar perovskite can be applied to adsorb probe molecules to obtain reproducible Raman scattering signals of biological molecules (DNA fragments and amino acids). More importantly, the charge transfer for SERS enhancement can be tuned by the single-atom sites, which can quantitatively transfer photoinduced charge and novel energy level for charge transfer. Additionally, considering the advantages of single-atom sites on chips, the uniform, low-cost and large-scale production of novel substrates is beneficial for practical SERS applications. Sensitive and quantitative SERS was successfully realized on portable Raman instruments. Thus, the single-atom site strategy with precise control of accessible sites on perovskite chips might open a new platform of sensitive and quantitative SERS for practical application, which is urgently needed in the communities of materials science, environmental science and biology.

\section{EXPERIMENTAL SECTION}

\section{Chemicals and materials}

All chemicals, including sodium tungstate dihydrate $\left(\mathrm{Na}_{2} \mathrm{WO}_{4} \cdot 2 \mathrm{H}_{2} \mathrm{O}\right)$, hydrochloric acid $(\mathrm{HCl})$, polyethylene glycol (molecular weight $M_{\mathrm{W}}=200 \mathrm{Da}$, abbreviated as PEG-200), ethanol, ethylene glycol (EG), lead bromide $\left(\mathrm{PbBr}_{2}\right)$, cesium bromide (CsBr), dimethyl sulfoxide (DMSO), rhodamine 6G 
(R6G), tyrosine (Tyr), and cytosine (Cyt) were obtained from Aladdin chemical reagent Corp (Shanghai, China) and employed without further purification. Water used in the experiments was Nanopure water made using a Barnstead Nanopure Diamond system.

\section{Synthesis of $\mathrm{CsPb}_{2} \mathrm{Br}_{5}$ (CPB) chip}

$\mathrm{PbBr}_{2}$ solution $\left(21 \mu \mathrm{L}, 1 \mathrm{~mol} \mathrm{~L}{ }^{-1}\right)$ was spin-coated on an indium tin oxide (ITO) chip at $2000 \mathrm{r} \mathrm{min}^{-1}$ for $30 \mathrm{~s}$ and dried on a hot plate at $100^{\circ} \mathrm{C}$ for $30 \mathrm{~min}$. After cooling to room temperature, $150 \mu \mathrm{L} \mathrm{CsBr}$ solution $\left(0.07 \mathrm{~mol} \mathrm{~L}^{-1}\right)$ was spin-coated on the chip at $2000 \mathrm{r} \mathrm{min}^{-1}$ for $30 \mathrm{~s}$ and dried on a hot plate at $250^{\circ} \mathrm{C}$ for $5 \mathrm{~min}$. By repeating this procedure 8 times, high-quality $\mathrm{CPB}$ chips were successfully synthesized on ITO.

\section{Synthesis of $\mathrm{W}_{1}-\mathrm{O}-\mathrm{CPB}$ chip}

The synthetic method of single tungsten site precursor is modified upon our previous work [40]. $\mathrm{Na}_{2} \mathrm{WO}_{4} \cdot 2 \mathrm{H}_{2} \mathrm{O}(2.27 \mathrm{mmol}$, $750 \mathrm{mg})$ and PEG-200 (1 mL) were dissolved in deionized water $(10 \mathrm{~mL})$, stirred for $30 \mathrm{~min}$ at ambient temperature, and then hydrochloric acid $(\mathrm{HCl})$ was added dropwise into the mixture until $\mathrm{pH}$ 5.5. The obtained solution was magnetically stirred in the dark for $6 \mathrm{~h}$. Finally, the as-synthesized CPB chip was first immersed in $30 \mathrm{~mL}$ of $\mathrm{EG} / \mathrm{H}_{2} \mathrm{O}$ (1:10) mixture, and then, $1200 \mu \mathrm{L}$ of the as-synthesized $\mathrm{W}$ single sites solution was added to the mixture. The mixture was sealed, heated at $60^{\circ} \mathrm{C}$ for $1 \mathrm{~h}$, and then annealed at $80^{\circ} \mathrm{C}$ for $12 \mathrm{~h}$.

\section{SERS experiments}

To study the SERS properties of these $\mathrm{W}_{1}-\mathrm{O}-\mathrm{CPB}$ chips, a confocal micro-Raman spectrometer (RENISHAW inVia) was used as the measuring instrument. In all SERS tests, unless specifically stated, the excitation wavelength was $532 \mathrm{~nm}$, the laser power was $0.5 \mathrm{~mW}$, and the specification of the objective was $50 \times \mathrm{L}$. A series of standard solutions of R6G, Tyr, and Cyt at different concentrations were used as the probe molecules. Synthesized $\mathrm{W}_{1}-\mathrm{O}-\mathrm{CPB}$ chips were immersed in $1 \mathrm{~mL}$ probe molecule solution at different concentrations for $1 \mathrm{~h}$. The probe@ $\mathrm{W}_{1}-\mathrm{O}-\mathrm{CPB}$ chips were washed with ethanol and water in an ultrasonic cleaner and dried at $60^{\circ} \mathrm{C}$. The portable Raman experimental conditions were as follows: laser wavelength: $785 \mathrm{~nm}$; power: $10 \mathrm{~mW}$; acquisition time: $10 \mathrm{~s}$.

\section{Characterization}

High-resolution transmission electron microscopy (HR-TEM), high-angle annular dark field scanning TEM (HAADF-STEM) and energy-dispersive X-ray spectroscopy (EDS) elemental mapping characterization were performed by a Titan Themis G2 transmission electron microscope operated at $300 \mathrm{kV}$ and equipped with a probe spherical aberration corrector. Inductively coupled plasma optical emission spectrometry (ICP-OES) measurements were conducted on an Agilent ICP-OES-730 analyzer.

\section{RESULTS AND DISCUSSION}

\section{Structural characterization of the single-atom sites on chip}

To achieve regular chips with controllable sites, we chose the well-investigated substrate of a lead halide perovskite (CPB) scintillator, which is widely investigated in solar cells and SERS due to its suitable absorption wavelength, highly engineered surface and excellent photon-to-electron conversion efficiency $[42,43]$. Then, single-atom sites can be controllably anchored on this substrate to obtain quantitative sites. We chose oxygencoordinated tungsten atom as the single-atom site, which can have 4-6 coordinated oxygen atoms surrounding the single tungsten atom. More importantly, the single-atom sites of oxygen-coordinated tungsten atoms have the advantage of highly efficient charge transfer [40]. A schematic of the synthesis process of single-atom sites on chips is shown in Fig. 1a. Photography of the flat and uniform chip is shown in Fig. 1a (right). An amplified sandwich-type structure of "ITO glass/perovskite/ single site" is demonstrated in the cross-section image via scanning electron microscopy (SEM) (Fig. 1b). After anchoring single sites on the perovskite, the perovskite surface remains smooth and neat, as characterized by SEM (Fig. 1c). Elemental mapping via EDS indicates that tungsten (W), cesium (Cs), lead $(\mathrm{Pb})$ and bromine $(\mathrm{Br})$ are uniformly dispersed on a partial piece of the chip at a scale of $10 \mu \mathrm{m}$ (Fig. 1d). Compared with pristine CPB (Fig. S1, Supplementary information), the SEM image of the single sites on perovskite chip $\left(\mathrm{W}_{1}-\mathrm{O}-\mathrm{CPB}\right)$ has no obvious morphological changes at $\mu \mathrm{m}$ scale. The X-ray diffraction (XRD) also shows that the single-atom sites would not induce a periodic lattice difference in perovskite (Fig. 1e). Moreover, to characterize this structure more precisely, the atomic-scale morphology of CPB was also verified by HR-TEM. Lattice distances of 0.268 and $0.300 \mathrm{~nm}$ represent the (310) and (220) facets of the CPB substrate, respectively (Fig. 1f). The singleatom sites of tungsten can be observed in the amplified image obtained by HAADF-STEM. The brightness contrast in Fig. $1 \mathrm{~g}$ shows that the tungsten atoms are anchored on a planar and thin $\mathrm{CPB}$ substrate. In addition, the single-atom sites precursor can also be identified by HAADF-STEM as shown in Fig. S2. Furthermore, the anchored single-atom sites on the perovskite can induce a valence state change in the substrate (Fig. S3) according to X-ray photoelectron spectroscopy (XPS). The $\mathrm{Pb}$ valence states have a $1.6 \mathrm{eV}$ upshift, which indicates that $\mathrm{Pb}$ (orthorhombic $\mathrm{Pb}-\mathrm{O}$ ) [44] has a stronger bonding with single sites than that in pristine $\mathrm{CsPb}_{2} \mathrm{Br}_{5}(\mathrm{~Pb}-\mathrm{Br})$. In contrast, the valence states of $\mathrm{W}$ and Cs exhibit no obvious changes (Fig. S3), and the 1:2 ratio of $\mathrm{W}^{5+} / \mathrm{W}^{6+}$ is consistent with that of as-prepared single sites [40]. These results suggest that the $\mathrm{Pb}$ atoms on the perovskite surface lattice might have bonded with oxygen atoms from the oxygen-coordinated single tungsten atom oxide.

To further analyze the configuration of a single-atom site more thoroughly, the $\mathrm{W}_{1}-\mathrm{O}-\mathrm{CPB}$ was further examined by $\mathrm{X}$ ray absorption near-edge structure (XANES) and extended Xray absorption fine structure (EXAFS). First, the Fourier-transformed (FT) EXAFS spectrum (Fig. 2a, b) shows one main peak of $\mathrm{W}-\mathrm{O}$ at $1.79 \AA$, in contrast to the $\mathrm{W}-\mathrm{W}$ coordination peak at $2.6 \AA$ shown in the $\mathrm{W}$ foil, confirming the existence of monodispersed atom sites. This is consistent with the HAADF-STEM result that the single-atom sites are randomly dispersed on the $\mathrm{CPB}$ surface. Furthermore, wavelet-transform EXAFS also indicates the distinct difference between single-atom sites and reference samples (Fig. $2 \mathrm{~d}-\mathrm{f}$ ). In addition, the $\mathrm{W} \mathrm{L}_{\mathrm{III}}$-edge XANES spectra were analyzed (Fig. 2c), in which the red-line peak of $\mathrm{W}_{1}-\mathrm{O}-\mathrm{CPB}$ is located nearby that of $\mathrm{WO}_{3}$, revealing that the presence of $\mathrm{W}^{5+}$ and mainly $\mathrm{W}^{6+}$, similar to the single-atom oxide precursor with a dominant chemical states of $6+$. Additionally, the coordinated number of single-atom sites on the perovskite is also steady. Thus, the anchoring process of $\mathrm{W}_{1}-\mathrm{O}-$ 

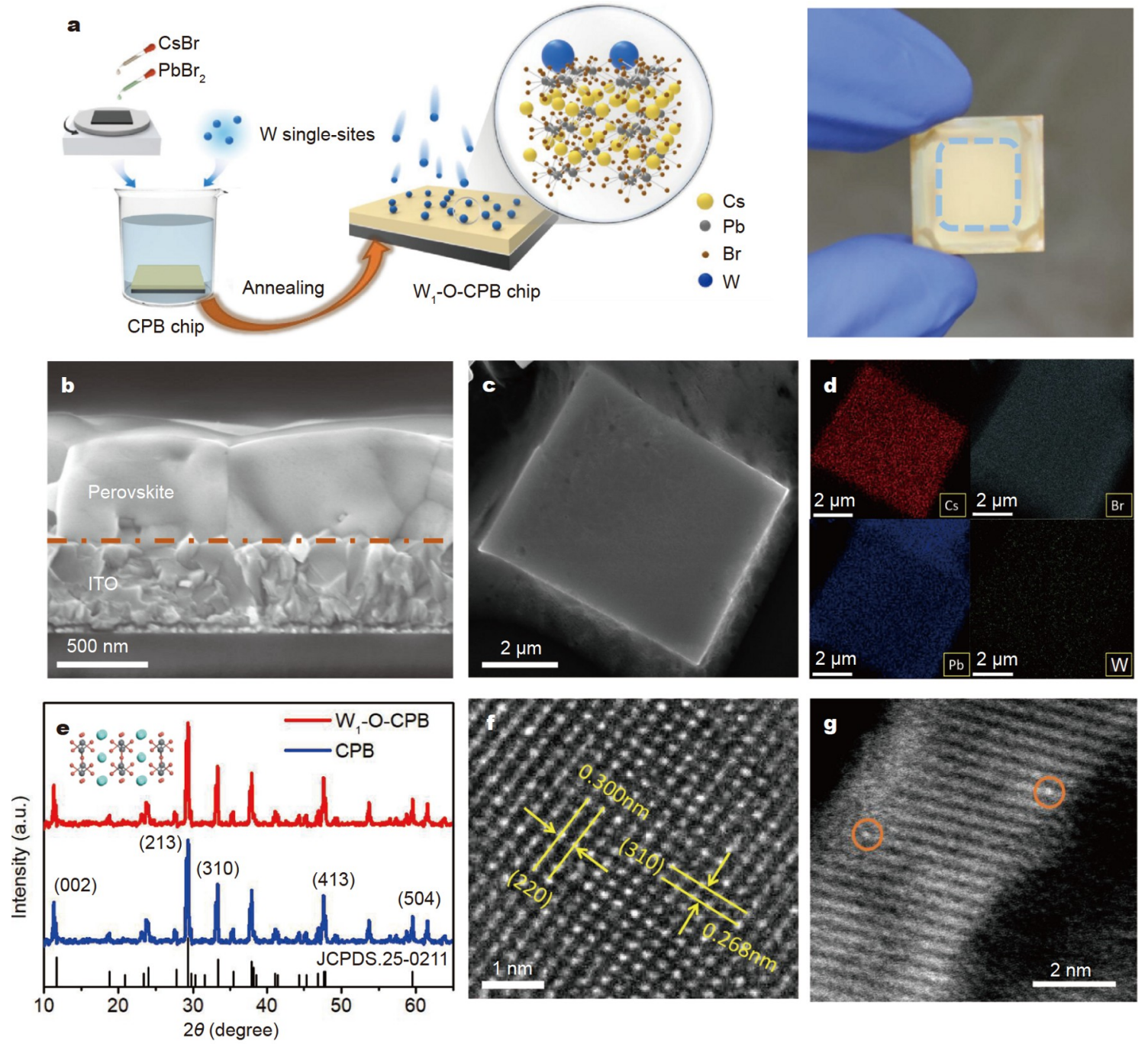

Figure 1 Synthesis and morphological characterization of the $\mathrm{W}_{1}-\mathrm{O}-\mathrm{CPB}$ chip. (a) Schematic illustration of the fabrication of the $\mathrm{W}_{1}-\mathrm{O}-\mathrm{CPB}$ chip (left). Photography of the $\mathrm{W}_{1}-\mathrm{O}-\mathrm{CPB}$ chip (right). The blue dotted zone of the chip is used for Raman detection. Blue balls represent oxygen-coordinated single tungsten atoms. (b) Cross-sectional SEM image demonstrating the perovskite film on ITO arrays. (c, d) SEM-EDS images of the $\mathrm{W}_{1}-\mathrm{O}-\mathrm{CPB}$ chip showing the uniform distributions of $\mathrm{Cs}, \mathrm{Pb}, \mathrm{Br}$ and $\mathrm{W}$ elements on the chip. (e) XRD patterns of CPB before and after $\mathrm{W}$ loading. (f, g) HAADF-STEM images revealing the lattice structure of the $\mathrm{W}_{1}-\mathrm{O}-\mathrm{CPB}$ chip and single sites on $\mathrm{CPB}$.

$\mathrm{CPB}$ has little impact on the single tungsten atom site. Combining with the XPS, EXAFS and TEM results, the configuration of $\mathrm{W}_{1}-\mathrm{O}-\mathrm{CPB}$ can be proposed to be as follows: the single-atom site is identified as $\mathrm{W}_{1}-\mathrm{O}-\mathrm{Pb}$, and the connecting oxygen should be the coordinated oxygen from single tungsten atom oxide. Compared with bare single tungsten atom oxide [40], the CPB substrate might induce a small distortion of the $\mathrm{W}_{1}-\mathrm{O}$ configuration (Fig. $2 \mathrm{a}$ and Table 1).

Sensitive SERS quantification by the single-atom sites on chip To demonstrate the SERS activity, a standard probe molecule $\left(\lambda_{\text {mol }} \approx \lambda_{\text {laser }}=532 \mathrm{~nm}\right)$, i.e., R6G, was first dissolved in different concentrations for characterization. The representative SERS spectra are shown in Fig. 3a. Furthermore, to further extend the single-atom sites on chip to a universal strategy for biological molecule detection, the aromatic amino acid Tyr and a single fragment of the molecule nucleotide Cyt were chosen for analysis (Fig. 3b, c). The quantitative analysis and the relative standard deviations (RSDs) are shown in Fig. $3 \mathrm{~d}-\mathrm{f}$ and $\mathrm{g}-\mathrm{i}$, respectively. In R6G Raman scattering, as shown in Fig. 3a, the R6G on $\mathrm{W}_{1}-\mathrm{O}-\mathrm{CPB}$ demonstrates an $\mathrm{R} 1$ peak at $612 \mathrm{~cm}^{-1}$ and an R2 peak at $772 \mathrm{~cm}^{-1}$ that can be clearly observed and assigned to in-plane and out-of-plane bending motions. Additionally, the R3 peak at $1362 \mathrm{~cm}^{-1}$ and R4 peak at $1651 \mathrm{~cm}^{-1}$ can be attributed to $\mathrm{C}-\mathrm{C}$ stretching vibrations of the aromatic nucleus. The significant peak at $612 \mathrm{~cm}^{-1}$ originating from $\mathrm{R} 6 \mathrm{G} @ \mathrm{~W}_{1}-\mathrm{O}-\mathrm{CPB}$ can be observed, as marked by the orange zone (Fig. 3a), to exhibit a linear increase as the R6G concentration increases (Fig. 3d), with a correlation coefficient of $R^{2}=0.997$. The linear limit of the single-atom sites on a chip can reach $10^{-9} \mathrm{~mol} \mathrm{~L}^{-1}$, which can be calculated as reaching a ultralow level of 60 molecules per spot according to the reported procedure [45]. The EF of R6G adsorbed on a single site was calculated to be as high as $10^{7}$ (see Supplementary information for calculation details), and a tunable EF is obtained at various probe concentrations as shown in Fig. S4. $\mathrm{W}_{1}-\mathrm{O}-\mathrm{CPB}$ has the record-high sensitivity among the plasmonic-free substrates and even is comparable to the electromagnetic mechanism (EM) dominant 

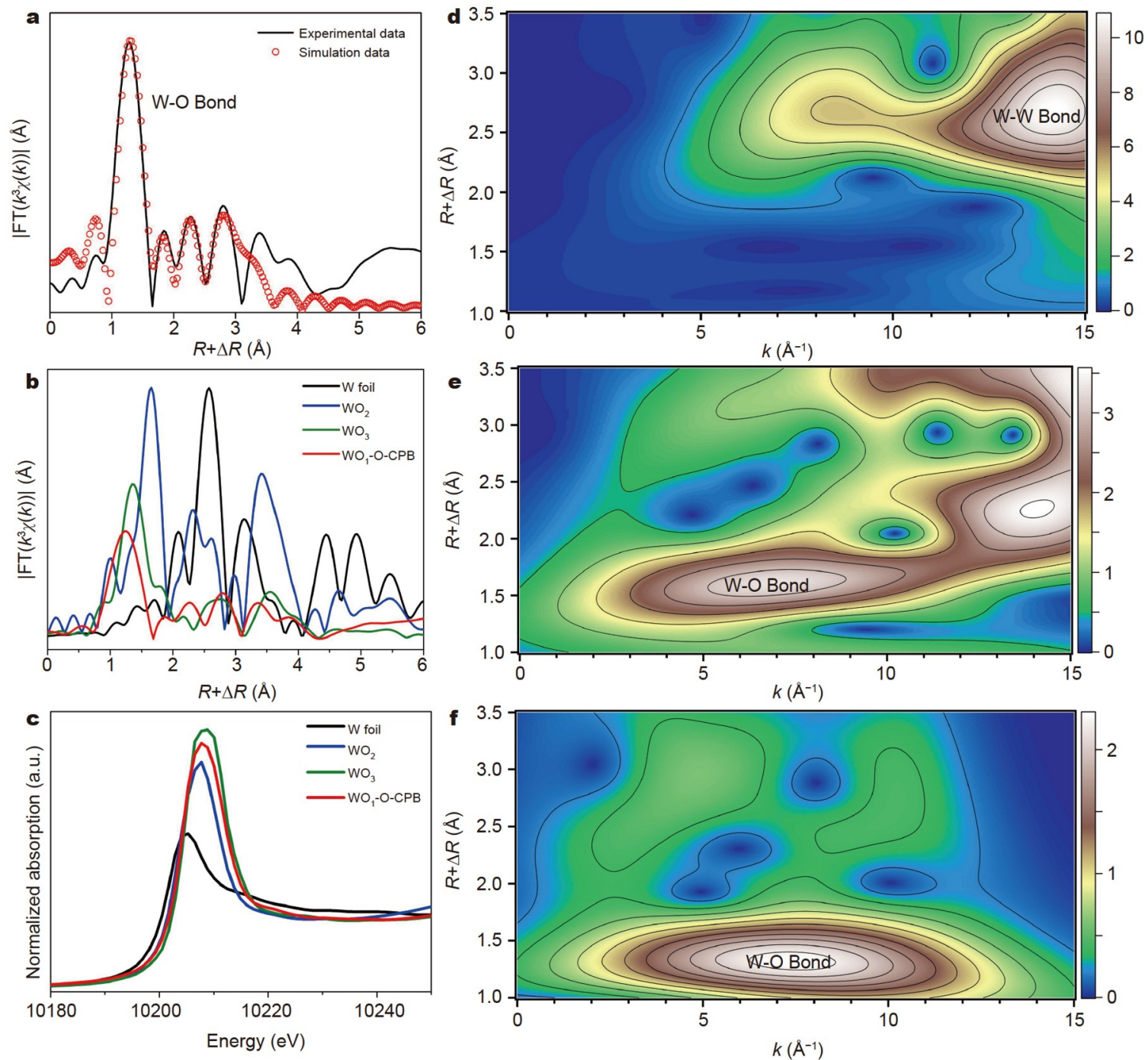

Figure 2 Atomic configuration of a single-atom site on the perovskite substrate. (a) Comparison of the FT-EXAFS curves between the experimental data and the fitted spectra of $\mathrm{W}_{1}-\mathrm{O}-\mathrm{CPB}$. (b, c) Corresponding $k^{3}$-weighted FT spectra of $\mathrm{W}_{1}-\mathrm{O}-\mathrm{CPB}, \mathrm{WO}_{2}, \mathrm{WO}_{3}$ and $\mathrm{W}$ foil: (b) the EXAFS spectra, (c) the $\mathrm{W} \mathrm{L}_{\mathrm{III}}{ }^{-}$ edge XANES spectra. The last three materials were used as reference samples. (d-f) Wavelet-transform EXAFS of the $\mathrm{W}_{1}-\mathrm{O}-\mathrm{CPB}$ and the reference samples of $\mathrm{W}$ foil and $\mathrm{WO}_{3}$ crystal.

Table 1 EXAFS fitting parameters at the $\mathrm{W} \mathrm{L}_{\mathrm{III}}$-edge for various samples $\left(S_{0}{ }^{2}=0.896\right)$

\begin{tabular}{ccccccc}
\hline Sample & Shell & $N^{\mathrm{a}}$ & $R(\AA)^{\mathrm{b}}$ & $\sigma^{2}\left(\AA^{2}\right)^{\mathrm{c}}$ & $\Delta E_{0}(\mathrm{eV})^{\mathrm{d}}$ & $R$ factor \\
\hline \multirow{2}{*}{$\mathrm{W}_{1}-\mathrm{O}-\mathrm{CPB}$} & $\mathrm{W}-\mathrm{O}$ & 2.6 & 1.79 & 0.0132 & 0.4 & 0.0241 \\
& $\mathrm{~W}-\mathrm{O}-\mathrm{Pb}$ & 2.1 & 2.81 & 0.0114 & & \\
\hline
\end{tabular}

a) $N$ : coordination number; b) $R$ : bond distance; c) $\sigma^{2}$ : Debye-Waller factor; d) $\Delta E_{0}$ : the inner potential correction. $R$ factor: goodness of fit. $S_{0}{ }^{2}$ was set to 0.896 , according to the experimental EXAFS fit of $\mathrm{W}$ foil by fixing $\mathrm{CN}$ as the known crystallographic value.

noble metals (single-particle condition). The previously reported detection limits in the literature are summarized in Fig. $4 \mathrm{~b}$ and Table S1. In addition, the reproducibility is confirmed by Raman mapping of R6G. One hundred runs in triplicate for SERS (Fig. 4a) on a single site provided visual $2 \mathrm{D}$ spectral mapping at a concentration of $10^{-7} \mathrm{~mol} \mathrm{~L}^{-1}$, and the RSD of $I_{612}$ was $4.5 \%$ (Fig. 3g), indicating obvious signal reproducibility and reliability. The reference samples selected for comparison are bare CPB, bare R6G and R6G@CPB. Fig. S5 illustrates the pristine CPB Raman scattering signals, showing that the inorganic substrate has several weak peaks from ethylene glycol surfactants, which is also confirmed by Fourier transform infrared (FTIR) spectroscopy indicating that the $-\mathrm{OH}$ group from the surfactant exists on the substrate surface, as shown in Fig. S6. To exclude the effect of molecules coordinated with single atoms, FTIR spectroscopy was performed, and the results (Fig. S7a) show that extra molecules on $\mathrm{CPB}$ have been completely removed by thermal treatment when the temperature reaches $400^{\circ} \mathrm{C}$ for $10 \mathrm{~h}$, which is below the phase-transition temperature of CPB. Fig. S7b exhibits similar Raman spectra of the sample before and after annealing treatment, indicating that the single-atom sites are contributed by the single metal atom sites rather than only 


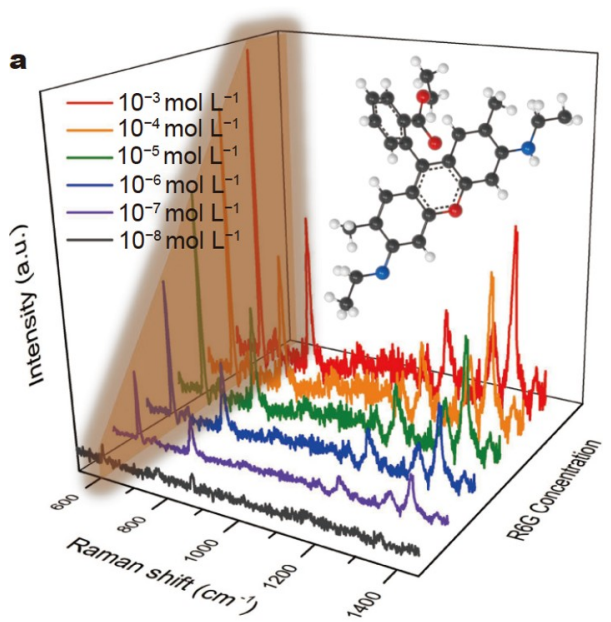

b
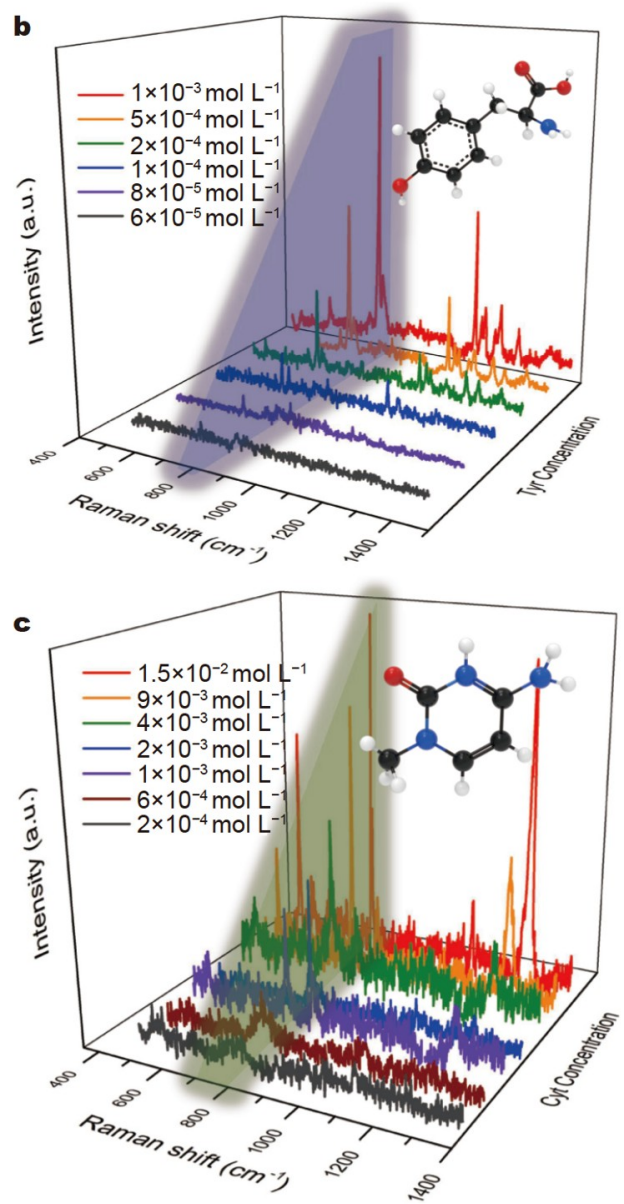
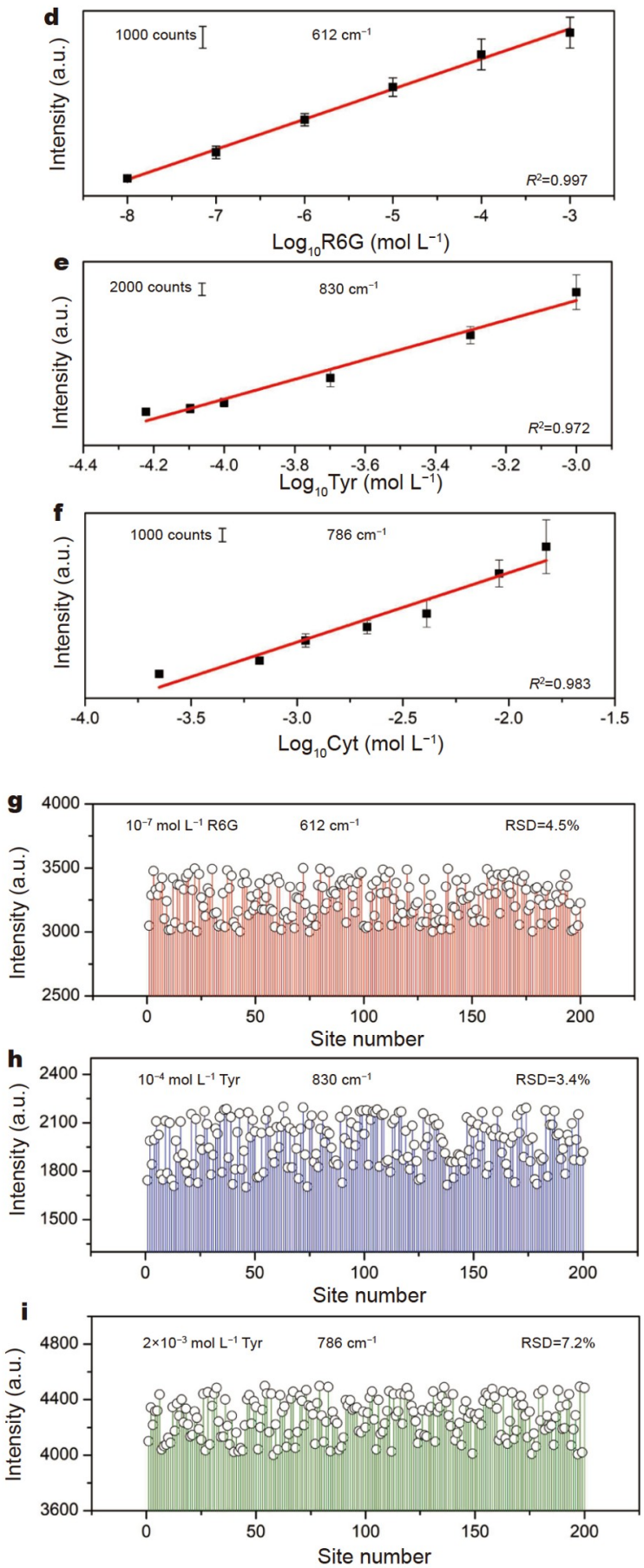

Figure 3 SERS properties of the $\mathrm{W}_{1}-\mathrm{O}-\mathrm{CPB}$ chip. $(\mathrm{a}-\mathrm{c})$ Gradually increasing Raman scattering signals recorded from the three analytes at different concentrations: (a) R6G, (b) tyrosine, (c) cytosine. Calibration curves of the peak intensity versus the logarithmic concentration of analytes: (d) R6G measured at $612 \mathrm{~cm}^{-1}$; (e) tyrosine measured at $830 \mathrm{~cm}^{-1}$; (f) cytosine measured at $786 \mathrm{~cm}^{-1}$. The top and bottom of the error bar represent the maximum and minimum

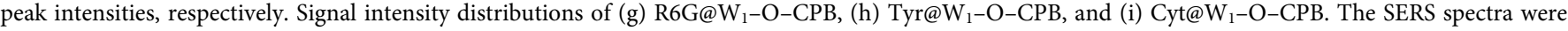
obtained by averaging three measurements from five randomly chosen spots.

by surface molecules. Furthermore, in contrast to the weak Raman scattering spectra of pristine $\mathrm{CPB}$ and bare R6G (Fig. S8a), the R6G@CPB (Fig. S8b) exhibits a high-intensity Raman signal, suggesting strong surface resonance Raman scattering. Surprisingly, when anchoring a single site to form R6G@W $\mathrm{W}_{1}-\mathrm{O}-\mathrm{CPB}$ (Fig. 3a and Fig. S8c), the single sites can effectively reduce the signal noise of background.
The sensitivity and quantification of nucleotides and amino acids also have similar performances. As shown in Fig. 3b, absorbed tyrosine exhibits a distinct characteristic Raman signal for the benzene-ring breathing doublet bending mode with an R1 peak at $860 \mathrm{~cm}^{-1}$ and an R2 peak at $830 \mathrm{~cm}^{-1}$. Notably, the intensity of the Raman peak at $830 \mathrm{~cm}^{-1}$ was used as a reference peak for further research. The asymmetric molecules of tyrosine 
cannot achieve the same limit of detection as R6G due to their relatively narrow Raman scattering cross-section and its electronic structure without resonance SERS, suggesting that more sensitivity of semiconductors should be further improved to detect these important biological molecules. Similar to that of R6G, the quantification of tyrosine is also illustrated in Fig. 3e. The linear increase in Raman intensity versus the log concentration of target tyrosine molecules from $60 \mu \mathrm{mol} \mathrm{L}^{-1}$ to $1 \mathrm{mmol} \mathrm{L}^{-1}$ with an $R^{2}$ of 0.972 reveals the capability for quantitative analysis. Cytosine could be able to be quantitatively detected from $200 \mu \mathrm{mol} \mathrm{L}{ }^{-1}$ to $15 \mathrm{mmol} \mathrm{L}^{-1}$ with an $R^{2}$ of 0.983 , as shown in Fig. 3c, f. Compared with bare tyrosine or cytosine
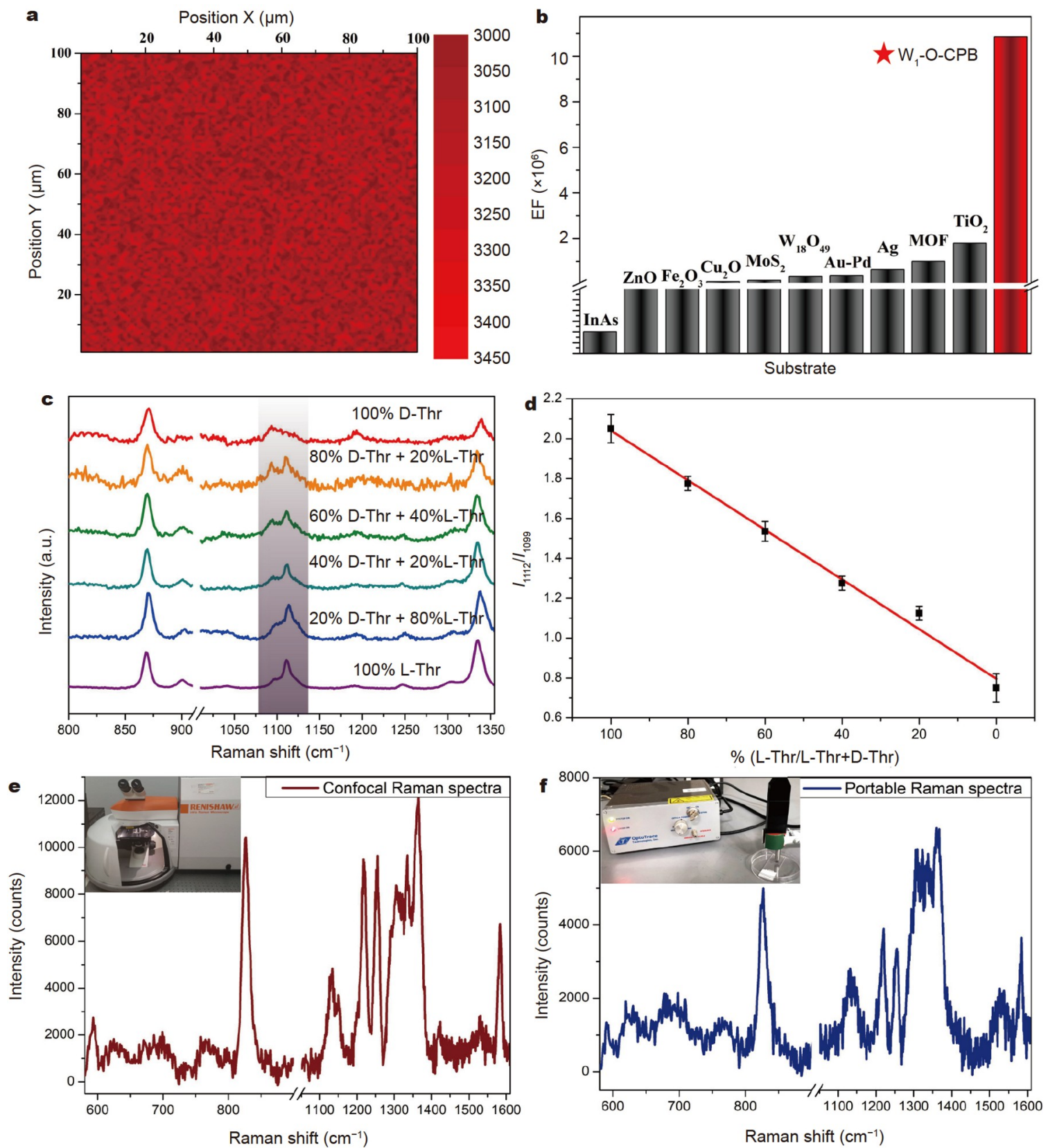

Figure 4 Stability and sensitivity of the $\mathrm{W}_{1}-\mathrm{O}-\mathrm{CPB}$ chip. (a) SERS mapping at $612 \mathrm{~cm}^{-1}$ of $10^{-7}$ mol L $\mathrm{L}^{-1} \mathrm{R} 6 \mathrm{G}$ adsorbed on the chip. (b) Comparison of the EFs between $\mathrm{W}_{1}-\mathrm{O}-\mathrm{CPB}$ chip and other substrates, including plasmon-free semiconductors and single-particle noble metals. (c) Normalized SERS spectra of the mixtures of $L$-Thr and $D$-Thr (the contents of $L$-Thr and $D$-Thr are changed from $100 \%$ to $0 \%$ ). (d) Calibration curve of the relative intensity ratio of $I_{1112} /$ $I_{1099}$ is plotted versus the content of $L$-Thr (\%) in the $L$ - and $D$-Thr mixture. (e, f) Comparison of tyrosine Raman spectra on $\mathrm{W}_{1}-\mathrm{O}-\mathrm{CPB}$ obtained via portable Raman and confocal Raman platforms (785 nm excitation). 
spectral discrepancies, which may be due to the tendency of chiral isomers. Surprisingly, plotting the difference in the ratio of the relative intensities of the peaks at $1112 / 1099 \mathrm{~cm}^{-1}$ against the $L$-Thr content (in \%) reveals a linear correlation with a coefficient of $R^{2}=0.989$.

Moreover, this single site on chip substrate can overcome the weaknesses of discrepancies of surface sites and low-quantumefficiency of photoinduced charges, so that it can be applied in portable Raman instruments. Fig. 4e, f show a comparison of the Raman spectra of Tyr $\left(10^{-3} \mathrm{~mol} \mathrm{~L}^{-1}\right)$ on $\mathrm{W}_{1}-\mathrm{O}-\mathrm{CPB}$ obtained via a portable Raman platform $(785 \mathrm{~nm})$ and a confocal Raman platform $(785 \mathrm{~nm})$. The similar limits of detection indicate that the uniform and highly efficient charge-transfer substrate can be broadly applied in practical Raman instruments. Additionally, the long-term stability of R6G on a single site was verified by the SERS detection after probe adsorption on chips for six months (Fig. S9), which might be ascribed to the chips effectively transferring the photoinduced holes away on ITO to avoid photooxidation [47]. Dispersed nanomaterials may have photoinduced analyte structural damage caused by photobleaching and/or metal-catalyzed side reactions, which is a severe drawback of the conventional SERS substrates [48]. Chips with layered structures can provide a promising approach for portable SERS activity in food safety, environmental protection and biological detection, which can balance the trade-off among the numerical aperture of spectrometers, the transmittance or reflectivity of the optical elements, and the noise of chargecoupled devices [49].

\section{SERS mechanism of the single-atom sites on chip}

Due to the plasmon-free nature of single-atom sites and CPB semiconductors, CMs are mainly considered, including charge generation, charge transfer and interaction between probes and sites, which are related to three features of single sites on chips: PL quenching, enhanced charge transfer and quantitative adsorption of probes.

\section{$P L$ quenching and enhanced charge-transfer mechanism}

Generally, photoinduced carriers are beneficial for SERS and the trapping of carriers from photoluminescent substrates can effectively reduce the noise caused by resonance Raman scattering [5]. Quantitative PL quenching and charge transfer are important issues in the photoinduced process. First, perovskite substrates are well known for their PL performance with ultrahigh photoelectron quantum efficiency (over 40\%), and it has been investigated as an SERS substrate with an EF of $10^{4-5}$ $[43,50]$. Fig. 5a demonstrates the gradually reduced PL intensity with the following sequence as: $\mathrm{CPB}, \mathrm{W}_{1}-\mathrm{O}-\mathrm{CPB}, \mathrm{R} 6 \mathrm{G} @ \mathrm{~W}_{1}-\mathrm{O}-$ $\mathrm{CPB}$. Moreover, the tunable PL quenching is shown as the density of single-atom sites (Table S2 measured by ICP-OES) increase as shown in Fig. 5b. The PL quenching can be quantified, showing that the quantum yield of charge transfer from $\mathrm{CPB}$ to single sites is approximately $\sim 66 \%$. The reduced PL is also beneficial for avoiding the resonance from the substrate PL, which is usually considered as the Raman noise. Regarding the R6G@CPB with a high resonance noise (Fig. S8b), the strategy of single-atom sites on perovskite chips can provide effective PL quenching, which results in not only elimination of background noise but also charge transfer. To further reveal the $\mathrm{PL}$ quenching in terms of kinetics, Fig. $5 \mathrm{c}$ shows that $\mathrm{W}_{1}-\mathrm{O}-\mathrm{CPB}$ has a shorter lifetime and a specific decay process compared with pristine $\mathrm{CPB}$ and $\mathrm{R} 6 \mathrm{G} @ \mathrm{CPB}$, whose lifetimes are summarized in Table 2. This suggests that single-atom sites on $\mathrm{CPB}$ provide new energy levels for charge transfer to adsorbed R6G.

To further confirm this charge transfer between $\mathrm{W}_{1}-\mathrm{O}-\mathrm{CPB}$ and the probe molecules, their interaction was measured by ultraviolet visible (UV-Vis) spectra as shown in Fig. $5 d$. The $\mathrm{W}_{1-}$ $\mathrm{O}-\mathrm{CPB}$ substrate has a similar absorption as pristine $\mathrm{CPB}$, and the R6G molecules on single-atom sites of $\mathrm{W}_{1}-\mathrm{O}-\mathrm{CPB}$ exhibit a 7 -nm redshift compared with that of bare R6G. This reveals that charge transfer between the single sites and probe molecules induced by the CM exists at single-atom sites on chip. Consistently, Fig. S8 also shows that the Raman peaks of R6G at single sites have a different spectrum compared with that of bare R6G, supplementarily indicating that the semiconductor-molecule interaction at the molecule/substrate interface was changed by single-atom sites. To further reveal the charge transfer and adsorption upon single-atom sites decoration on CPB, density functional theory (DFT) calculations were performed to investigate the spatial charge redistributions between the R6G molecule and $\mathrm{CsPb}_{2} \mathrm{Br}_{5}$ (001), as shown in Fig. 5e-g. The possible configurations were considered as a single site coordinated with $\mathrm{Pb}$ atoms, as shown in Fig. 5e. Clearly, the charge redistribution is more remarkable on modified surfaces $\left(0.26 \mathrm{e}^{-}\right.$from $\mathrm{W}_{1}-\mathrm{O}-\mathrm{CPB}$ to $\mathrm{R} 6 \mathrm{G} v$ s. $0.16 \mathrm{e}^{-}$from bare $\mathrm{CPB}$ to $\left.\mathrm{R} 6 \mathrm{G}\right)$, which is consistent with experimental evidence of increased molecular polarizability. Furthermore, the plane-averaged electron density of CPB before and after single-atom site decoration is visualized in Fig. S10b, which might be induced by the distortion of CPB upon single-atom site anchoring. Hence, incorporation of single-atom sites gives rise to remarkable charge increase at the surface region, implying a higher ability to donate charges to the molecule. Thus, the calculations clearly demonstrated that the unique advantage of single-atom sites is effective improvement of the interfacial charge transfer and thus the magnified molecular polarization.

Notably, in addition to the static coupling of the analyte and single-atom site, to directly verify highly efficient charge transfer from single sites to probe, photocarrier kinetics were studied by a transient absorption technique by probing the nonresonant photon intensity induced by a laser pulse with a central wavelength of $530 \mathrm{~nm}$. Fig. 6a shows the obtained transient absorption decay spectra of various samples $\left(\mathrm{CPB}, \mathrm{W}_{1}-\mathrm{O}-\mathrm{CPB}\right.$, R6G@W $1-\mathrm{O}-\mathrm{CPB}$ ), and the exciton decay follows the bi-exponential model with $\tau_{1}$ (fast decay) and $\tau_{2}$ (slow decay) (detailed data in Table 3). The short time constant of $\tau_{1}(0.89$ and $13.44 \mathrm{ps})$ can be attributed to fast processes associated with exciton formation: the free carriers injected by the pump pulse can induce a differential reflection signal more effectively than the excitons through the screening effect. Hence, the slow decay of $\tau_{2}$ is attributed to the exciton lifetime. Compared with pristine $\mathrm{CPB}$, the single-atom site on the $\mathrm{CPB}$ chip can significantly extend the lifetime from 22.99 to 83.16 ps, which indicates that single-atom sites can effectively trap the photoinduced electrons from $\mathrm{CPB}$. Interestingly, when probe $\mathrm{R} 6 \mathrm{G}$ is adsorbed onto the single sites, the lifetime is remarkably reduced to $0.52 \mathrm{ps}$, suggesting that the trapped electron can be transferred to probe molecules through the single-atom site pathway. Moreover, the photoinduced electron transfer process was analyzed based on Fig. $6 \mathrm{~b}$ and Table 3 . First, the red circles show that the signal in $\mathrm{W}_{1}-\mathrm{O}-\mathrm{CPB}$ rapidly rises. The red curve over the data represents the integral of a Gaussian function with a full width at half 

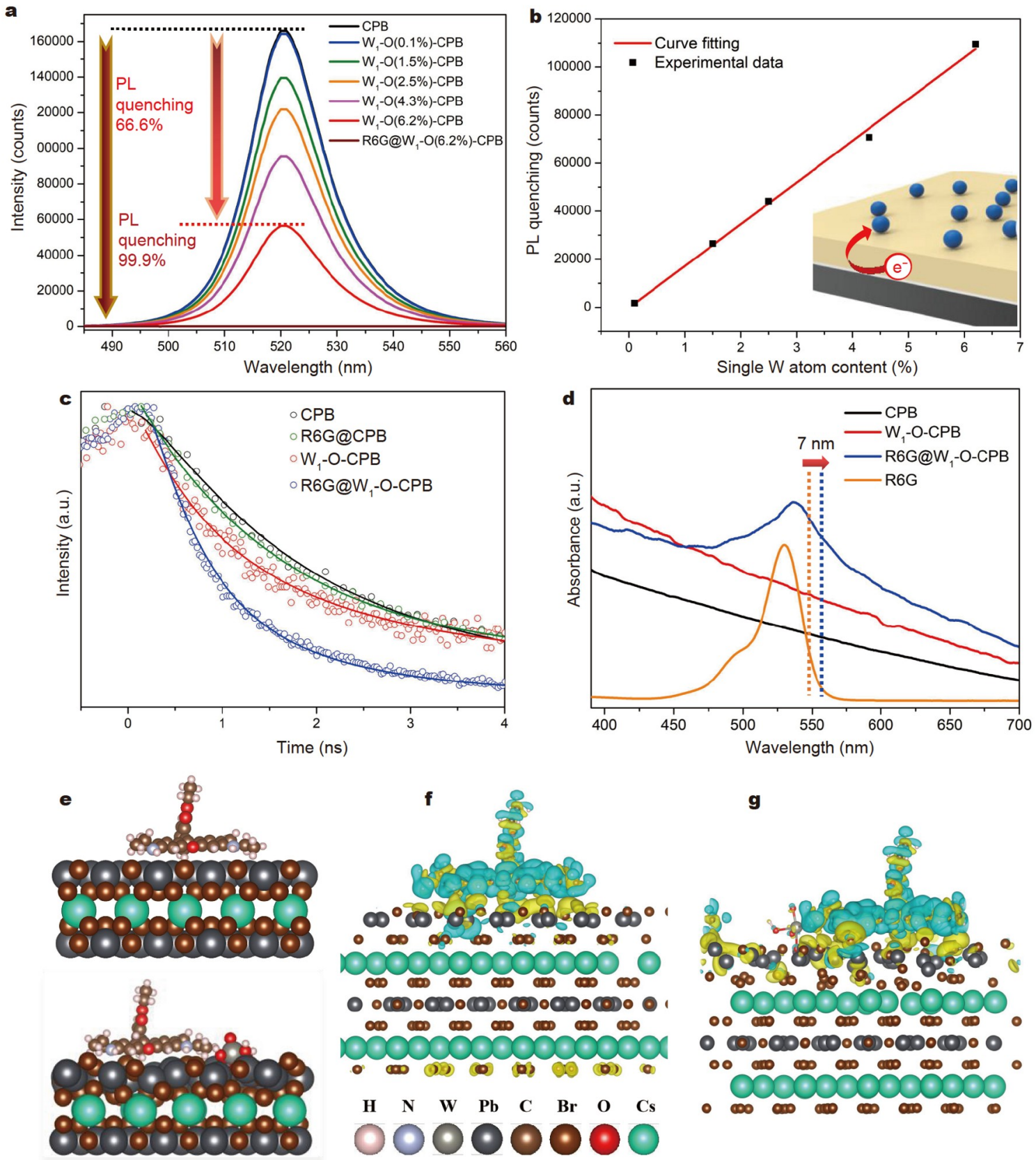

Figure 5 Mechanism of the absorption and charge-transfer process on the $\mathrm{W}_{1}-\mathrm{O}-\mathrm{CPB}$ chip. (a) PL spectra of $\mathrm{W}_{1}-\mathrm{O}-\mathrm{CPB}$ with various single-atom sites density (0-6.2 wt\%) and that of R6G@ $@ \mathrm{~W}_{1}-\mathrm{O}-\mathrm{CPB}$. (b) Linear relationship between PL quenching counts and single-atom sites density. Inserted schematic shows the charge transfer from $\mathrm{CPB}$ to a single $\mathrm{W}$ site. (c) Transient PL spectra of R6G@W $\mathrm{W}_{1}-\mathrm{O}-\mathrm{CPB}$ compared with $\mathrm{W}_{1}-\mathrm{O}-\mathrm{CPB}, \mathrm{R} 6 \mathrm{G} @ \mathrm{CPB}$ and bare $\mathrm{CPB}$. (d) UV-Vis absorption spectra of R6G@W $\mathrm{W}_{1}-\mathrm{O}-\mathrm{CPB}$ compared with R6G. (e) Side view of configuration of R6G@CPB and R6G@W simulation. The green, dark gray, brown, light gray, red and pink balls represent $\mathrm{Cs}, \mathrm{Pb}, \mathrm{Br}, \mathrm{W}, \mathrm{O}$ and $\mathrm{H}$ atoms, respectively. (f) Charge density difference for R6G adsorption on pristine $\mathrm{CPB}$, and $(\mathrm{g})$ single-atom site modified $\mathrm{CsPb}_{2} \mathrm{Br}_{5}(001)$. Yellow and cyan regions correspond to charge accumulation and depletion, respectively. The iso-surface value is 0.0003 electrons $\AA^{-3}$.

Table 2 Parameters settings and data analysis of transient PL spectra (excitation wavelength: $395 \mathrm{~nm}$, emission wavelength: $520 \mathrm{~nm}$ )

\begin{tabular}{ccc}
\hline Sample & Exposure time $(\mathrm{s})$ & $\tau(\mathrm{ns})$ \\
\hline $\mathrm{CPB}$ & 102.48 & 1.686 \\
$\mathrm{~W}_{1}-\mathrm{O}-\mathrm{CPB}$ & 494.89 & 1.285 \\
R6G@CPB & 127.64 & 1.597 \\
R6G@W 1 -O-CPB & 131.24 & 0.593 \\
\hline
\end{tabular}

maximum of $0.7 \mathrm{ps}$. Such an ultrafast transfer can be ascribed to a newly formed energy level induced by the single-atom sites, in which subpicosecond interlayer charge transfer is generally obtained. Since the charge transfer time is much longer than the photoinduced electron lifetime, the majority of the electrons excited in $\mathrm{CPB}$ are effectively transferred to the single-atom sites. Furthermore, when $\mathrm{R} 6 \mathrm{G}$ is adsorbed on $\mathrm{W}_{1}-\mathrm{O}-\mathrm{CPB}$, the rising time corresponds to a width of $0.43 \mathrm{ps}$, as shown by the blue- 

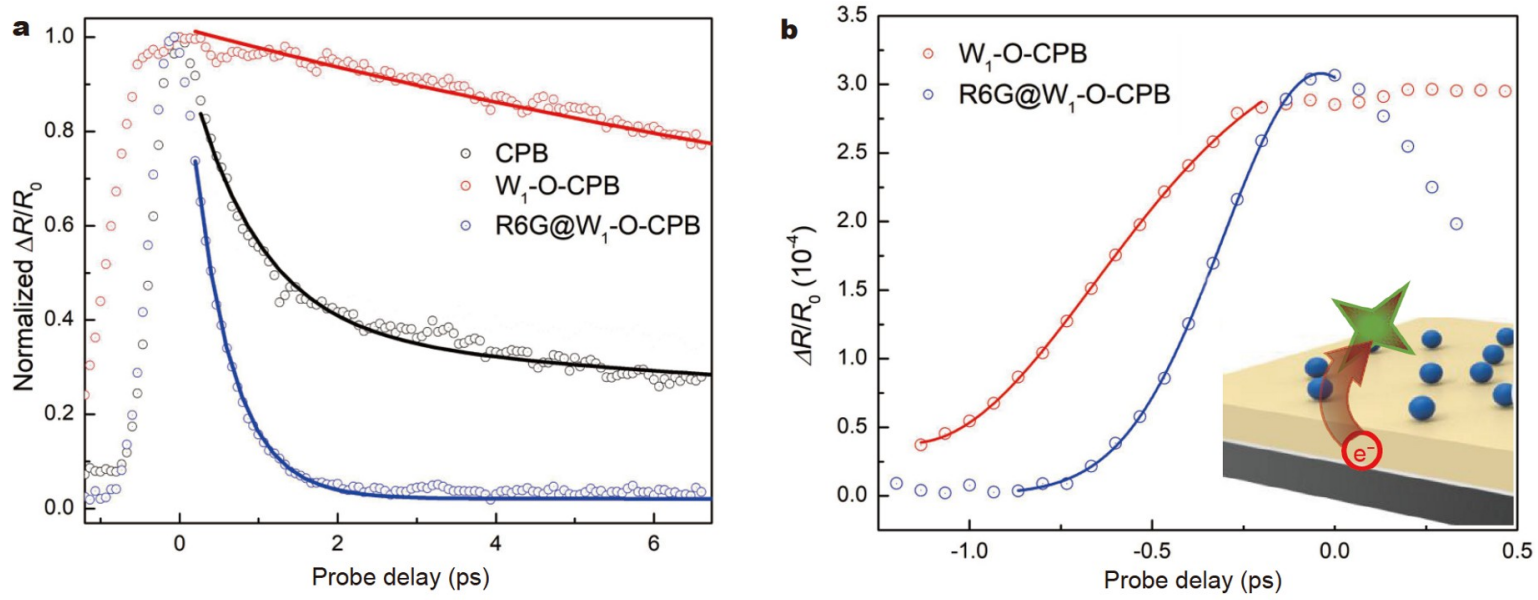

c

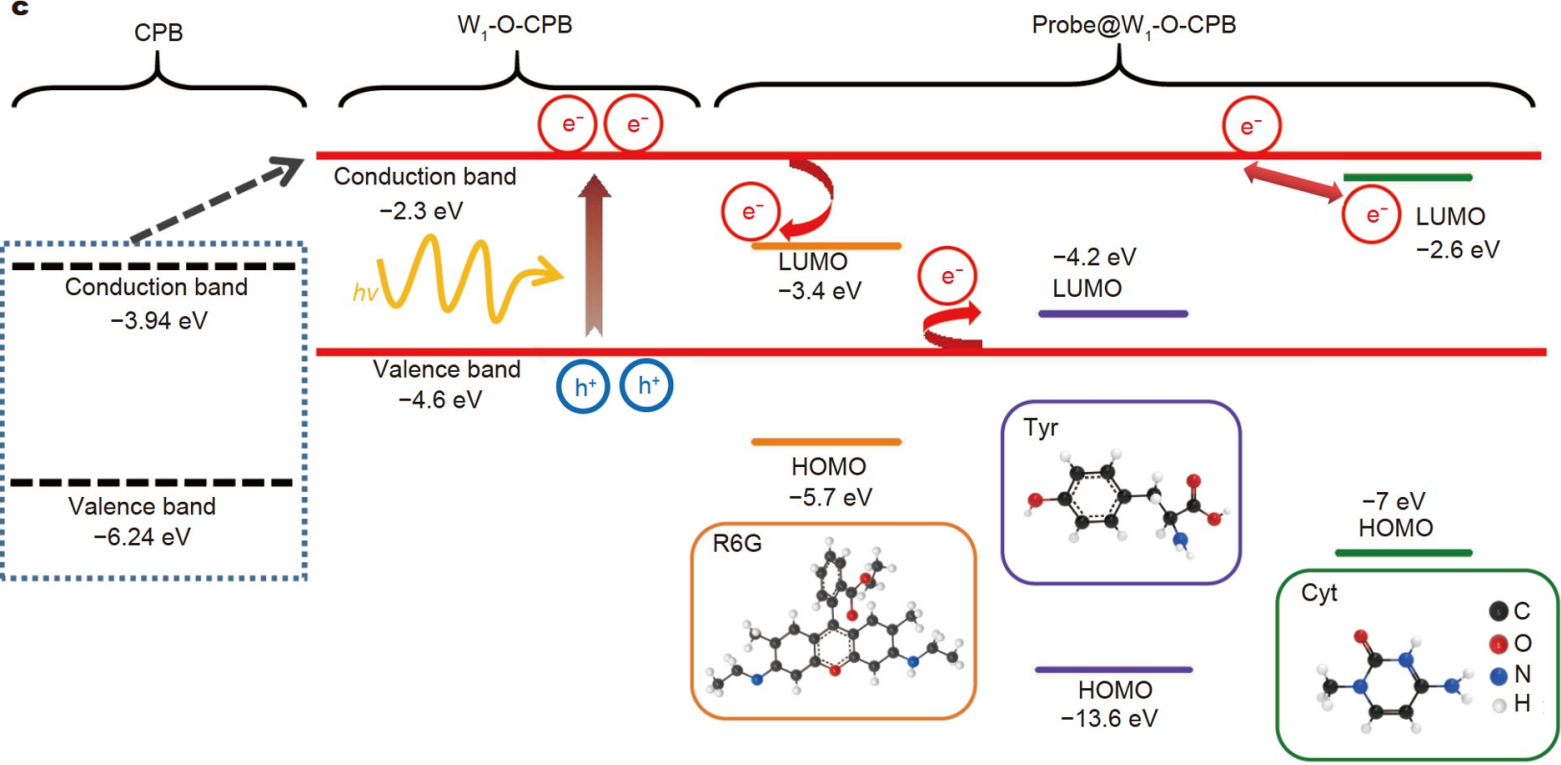

Figure 6 Photoinduced charge transfer of single-atom site on perovskite. (a) Transient absorption spectra of R6G@ $\mathrm{W}_{1}-\mathrm{O}-\mathrm{CPB}$ compared with $\mathrm{W}_{1}-\mathrm{O}-\mathrm{CPB}$ and CPB. (b) The blue curve and red curve are the fitting to the charge-induced transfer processes of $\mathrm{W}_{1}-\mathrm{O}-\mathrm{CPB}$ and $\mathrm{R} 6 \mathrm{G} @ \mathrm{~W}_{1}-\mathrm{O}-\mathrm{CPB}$, with transfer times of 0.70 and $0.43 \mathrm{ps}$, respectively. Inserted schematic shows the charge transfer between CPB and the single site with analyte molecules. (c) Schematic illustration of the energy levels and photoinduced charge transfer of pristine $\mathrm{CPB}, \mathrm{W}_{1}-\mathrm{O}-\mathrm{CPB}, \mathrm{R} 6 \mathrm{G}$, tyrosine, and cytosine.

circled curve in Fig. 6b, which suggests that the electron transfer on the R6G@ $\mathrm{W}_{1}-\mathrm{O}-\mathrm{CPB}$ is faster than that on $\mathrm{W}_{1}-\mathrm{O}-\mathrm{CPB}$. This may be due to that the formation of new electronic states on single-atom sites may interact with lowest unoccupied molecular orbital (LUMO) of the probe molecule, where electrons take a new pathway in the transfer process. This result is consistent with the transient PL results (Fig. 5c). Combining with these steady-state (PL and absorption) and time-resolved (PL and absorption) spectra, direct evidence of charge transfer from the $\mathrm{CPB}$ substrate to single-atom sites and further to probe molecules is presented, resulting in much enhanced SERS activity. Moreover, the quantum yield of charge transfer via single-atom sites can be revealed, which involves not only quenching of $\sim 66 \%$ of photoinduced carriers in steady-state but also shows an areal carrier density from single-atom sites to analytes as summarized in Table 3.

The corresponding band structure (Fig. 6c) of single-atom sites on perovskite indicated that the photoinduced charge transfer involves PL quenching of the substrate and charge transfer to the probe molecule at the single-atom sites or nearby. The photoinduced charge transfer sequence is " $\mathrm{CPB}$-singleatom site-analyte": (i) first, photoinduced electrons are generated from the perovskite substrate; (ii) then, they are transferred to single-atom sites; (iii) finally, they interact with the target molecules. The unique photoinduced charge transfer relies on a single-atom site with high charge-transfer capability. Moreover, according to the Herzberg-Teller selection rule, charge-transfer processes resonant with incident light selectively enhance different modes of probe molecules, and quantitative charge transfer might determine the quantitative signals based on the specific symmetric vibrational modes of probe molecules [51]. Compared with absorption under $532 \mathrm{~nm}$ excitation, an obvious decline $(\sim 44 \%)$ in the Raman intensity was observed under $633 \mathrm{~nm}$ irradiation (Fig. S11), while small Raman signals were obtained under $785 \mathrm{~nm}$ (Fig. S11). The results indicate that specific resonance from the band diagram plays an important role in Raman enhancement, and the Raman spectra of the adsorbed molecules strongly depend on the energy of the inci- 
Table 3 Parameters settings and data analysis of the transient absorption spectra

\begin{tabular}{ccccc}
\hline Sample & $\tau_{1}(\mathrm{ps})$ & $\tau_{2}(\mathrm{ps})$ & $\begin{array}{c}\text { Absorption coefficient } \\
\left(\mathrm{m}^{-1}\right)\end{array}$ & $\begin{array}{c}\text { Areal carrier density } \\
\left(\times 10^{14} \mathrm{~m}^{-2}\right)^{\mathrm{a}}\end{array}$ \\
\hline CPB & $0.887 \pm 0.031$ & $22.992 \pm 0.618$ & $283,410.1$ & $\begin{array}{c}\text { Charge transfer } \\
\text { time }(\mathrm{ps})^{\mathrm{b}}\end{array}$ \\
$\mathrm{W}_{1}-\mathrm{O}-\mathrm{CPB}$ & $13.441 \pm 0.450$ & $83.164 \pm 4.578$ & $248,847.9$ & 3.4829 \\
$\mathrm{R} 6 \mathrm{G} @ \mathrm{~W}_{1}-\mathrm{O}-\mathrm{CPB}$ & - & $0.52597 \pm 0.005$ & $248,847.9$ & 2.940 \\
\hline
\end{tabular}

a) Carrier density calculation details are given in the Supplementary information; b) charge transfer data are shown in Fig. S13. Pump wavelength 410 nm, probe wavelength $520 \mathrm{~nm}$.

dent light. To illustrate the charge-transfer process in terms of the thermodynamics, a band structure is drawn containing bare $\mathrm{CPB}, \mathrm{W}_{1}-\mathrm{O}-\mathrm{CPB}$ and probe molecules (Fig. $6 \mathrm{c}$ and Fig. S12). The locations of the valence band maximum (VBM) of CPB and $\mathrm{W}_{1}-\mathrm{O}-\mathrm{CPB}$ were measured, and their energy levels of $\mathrm{VBM}$ are -6.24 and $-4.6 \mathrm{eV}$, respectively (Fig. S12a-c). According to the UV-Vis absorption spectra (Fig. S12b, c), the bandgaps of CPB and $\mathrm{W}_{1}-\mathrm{O}-\mathrm{CPB}$ are approximately near $2.3 \mathrm{eV}$, and their conduction band minimum (CBM) levels should be at -3.94 and $-2.3 \mathrm{eV}$. This is consistent with the simulated density of states of $\mathrm{W}_{1}-\mathrm{O}-\mathrm{CPB}$ having an upshift compared with that of $\mathrm{CPB}$ (Fig. S10a). Together with previously reported data on R6G, amino acids and nucleobases [52], the highest occupied molecular orbital (HOMO) and LUMO for R6G, tyrosine, and cytosine are $-5.7 /-3.4,-13.6 /-4.2$ and $-7.0 /-2.6 \mathrm{eV}$, respectively. Hence, the charge transfer is illustrated as red arrows in Fig. 6c. This result is also consistent with $\mathrm{W}_{1}-\mathrm{O}-\mathrm{CPB}$ having an electron trapping capability and a lower energy level than the $\mathrm{CBM}$ of $\mathrm{CPB}$.

According to the Herzberg-Teller vibrational coupling law, molecular resonances caused by the photoinduced charge transfer greatly enhance the polarization tensor of molecules, correspondingly increasing the Raman scattering cross-section. Given that the wavelength of the excitation lasers used in Raman measurement was $532 \mathrm{~nm}$, which is close to the PL peak of the $\mathrm{CPB}$ substrate, the high EF value would be strongly related to the photoinduced enhancement effect of the CPB substrate $\left(\lambda_{\text {sub }} \approx\right.$ $\left.\lambda_{\text {laser }}\right)$. In addition, excited electrons can also be induced by probes. For the R6G molecule, whose bandgap is very close to the excitation wavelength $(532 \mathrm{~nm})$, resonance SERS also contributes to the charge-transfer process [19]. The three resonances (molecular resonance, photoinduced charge transfer and charge transfer in semiconductors) involved in SERS do not work independently. Consistently, as shown in Fig. S11, when the excitation wavelength is changed from 532 to $633 \mathrm{~nm}$, the relative intensities of the 612 and $773 \mathrm{~cm}^{-1}$ peaks of R6G considerably decrease, suggesting that the dominant charge transfer induced by $633 \mathrm{~nm}$ irradiation $(1.95 \mathrm{eV})$ is different from that induced by $532 \mathrm{~nm}$ irradiation. This might result in higher limit of detection concentrations for nucleobases and amino acids with larger bandgaps.

\section{Single-atom site quantification mechanism}

The quantification mechanism of single-atom sites on chips is revealed to involve two main aspects: quantitative sites and probe adsorption on the site or nearby. The quantitative amount of single sites on chips can be tuned from 0 to $\sim 6 \mathrm{wt} \%$ through alteration of the anchoring of single-atom sites, as confirmed by ICP-OES as shown in Table S2. Under the condition of $\sim 6 \mathrm{wt} \%$ loading of single-atom sites, the coverage of sites can also be calculated as $7.647 \times 10^{8}$ per $7.85 \times 10^{-13} \mathrm{~m}^{2}$ (within a laser spot, detailed in Supplementary information), indicating that the number of capture sites is sufficient for analyte detection at single molecule level. Further addition of single-atom sites would cause the rough morphology and distortion of the perovskite chip (Fig. S13a-c) compared with the smooth surface (Fig. 1b-c). The surface variation of sites might result in poor reproducibility. Meanwhile, the adsorption is essentially driven by electrostatic Coulomb force between the surface areas with single-atom sites and probe molecules due to their distinctively positive and negative charges (Table S3). Fig. S14a shows a uniform dispersion of R6G on single-atom sites anchored chip, whereas the pristine chip with the same concentration of R6G has obviously lower loading, as shown in Fig. S14b, suggesting that the single sites could act as capture sites on the surface. Fig. S14a-d also show a gradual increase in adsorbed R6G as the density of single-atom sites increases. Table S3 further verifies the positive zeta potentials of various molecules, and the negative zeta potential of $\mathrm{W}_{1}-\mathrm{O}-\mathrm{CPB}$ gradually increases as the single-atom sites loading amount increases on the perovskite. The reason might be that single-atom sites can form -OH on the surface to exhibit negative potentials. Thus, as the probe molecules in certain $\mathrm{pH}$ environments can exhibit positive potentials, the perovskite with the highest loading of single-atom sites exhibits the largest quantitative range in SERS activity (Fig. S15). Additionally, the variations in the charge transfer amount for different molecules can also be ascribed to various electrophilic abilities of functional groups [53].

According to the classic adsorption model [54], the relationship between the concentration $(C)$ in the system and the number $(N)$ of adsorbents on the surface exhibits a " $\log (C-N)$ " quasi-linear curve following the Freundlich adsorption isotherm: $q=k_{\mathrm{f}} C^{n}$, where $q$ is the adsorption density, $C$ is the dissolved concentration of the adsorbate, and $k_{\mathrm{f}}$ and $n$ are empirical constants. For the Freundlich isotherm, the log-log version is used: $\log q_{\mathrm{e}}=\log k_{\mathrm{f}}+1 / n \log C$.

Hence, we chose the highest loading of single-atom sites to detect Raman scattering of the various molecules, including dyes, amino acids and DNA fragments. The Raman scattering signals of Fig. $3 \mathrm{~d}-\mathrm{f}$ all exhibit a quasi-linear relation with $\log C$, suggesting that the adsorbed molecules near the sites are involved in the resonance Raman intensity $(I)$. The " $\log (C-(N)$ I)" relationship is also consistent with other quantitative studies on dye probe molecules. Additionally, the experimental adsorption is demonstrated to be physical adsorption on sites via multiple-time and long-term washing experiments (see Supplementary information), showing that the Raman signals of R6G have no obvious changes (Fig. S16). Hence, the strategy of single-atom sites on chip can provide quantitative capture sites, which is a universal approach through nonspecific Coulomb 
force following the nonselective adsorption rule in SERS.

\section{Discussion}

The substrate materials in SERS are indispensable for ultrasensitive detection of biomolecules. Our experimental results clearly demonstrated that single-atom sites enabled ultrahigh sensitivity to biomolecules, which can be ascribed to the charge transfer capability and the favorable energy level, leading to the significant magnification of molecular polarization and remarkable enhancement of Raman scattering. Notably, photoinduced electron separation of the perovskite materials can realize nearly $100 \%$ quantum efficiency, which is much higher than those of other semiconductors. Thus, the perovskite materials are great candidates for this strategy. Recently, perovskites have been studied to achieve a higher EF $[43,50]$. Our strategy of single-atom sites on chips provides a new route to discover various sites with satisfactory localized charges to achieve robust sensitive SERS with various extensions, including portable Raman detection for POCT/IVD. The single-atom sites on chips also exhibit quantitative and reproducible SERS detection, which is determined by controllable sites with new mechanisms. The advantage of single-atom sites on chip can be summarized as (1) generating sufficient charge from high-efficiency PL substrate to probe molecules, (2) providing a chargetransfer path, and (3) offering certain active sites for adsorption. Meanwhile, the SERS quantification on other semiconductors should have the similar features. For example, MOFs and carbon-based organic semiconductors $[25,28,29,55]$ have served as successful candidates with the advantages of high-density capture sites and quantitative interactions. Nevertheless, the limit of detection is still far from the noble metal hotspot-based substrates, and the mechanism of the adsorption is usually missing, which is a prerequisite for quantification. Single-atom sites strategy can give more precise configurations to study the adsorption model, such as a single layer or a single cluster. Single-atom sites prove that the sites can control the site density, site electrophilicity and electronic states, which should be further investigated to achieve a more suitable substrate for biological molecule detection.

\section{CONCLUSIONS}

High sensitivity and reproducible quantification in SERS detection are successfully achieved by modifying a single-atom site of tungsten atom oxide on lead halide perovskite chips. Through the noble-metal-free single-site strategy, quantitative detection at the ultralow level was successfully realized for R6G down to $10^{-9} \mathrm{~mol} \mathrm{~L}^{-1}$, which is more sensitive than plasmon-free semiconductors in the literature by at least one order of magnitude. More importantly, a single site on a chip can provide quantitative linear SERS responses from $60 \mu \mathrm{mol} \mathrm{L}^{-1}$ to $10 \mathrm{mmol} \mathrm{L}^{-1}$ (saturated) for biological amino acid detection and from $200 \mu \mathrm{mol} \mathrm{L}^{-1}$ to $45 \mathrm{mmol} \mathrm{L}^{-1}$ for nucleotide detection, which are the highest EFs among semiconductors to the best of our knowledge. The mechanism of superior SERS is ascribed to the controllable single sites, which not only provide quantitative sites for analyte adsorption but also enhance the charge transfer by effectively trapping photoinduced electrons from the substrate and transporting them to the analytes. Consistently, the PL quenching and charge-transfer sequence of single-atom sites is revealed to be "perovskite-single-atom site-analyte" by simulation and experimental results. Moreover, this highly efficient substrate has a result on a portable Raman instrument similar to that on a benchtop confocal Raman instrument due to the sufficient photoinduced charge density. Therefore, a sensitive SERS quantification platform can be built based on the strategy of single-atom sites on chips, which paves the way for novel material design for SERS in IVD and POCT.

\section{Received 11 January 2022; accepted 12 January 2022;} published online 2 March 2022

1 Cardinal MF, Vander Ende E, Hackler RA, et al. Expanding applications of SERS through versatile nanomaterials engineering. Chem Soc Rev, 2017, 46: 3886-3903

2 Garcia-Rico E, Alvarez-Puebla RA, Guerrini L. Direct surface-enhanced Raman scattering (SERS) spectroscopy of nucleic acids: From fundamental studies to real-life applications. Chem Soc Rev, 2018, 47: 49094923

3 Bell SEJ, Charron G, Cortés E, et al. Towards reliable and quantitative surface-enhanced Raman scattering (SERS): From key parameters to good analytical practice. Angew Chem Int Ed, 2020, 59: 5454-5462

4 Wang X, Huang SC, Hu S, et al. Fundamental understanding and applications of plasmon-enhanced Raman spectroscopy. Nat Rev Phys, 2020, 2: 253-271

5 Langer J, Jimenez de Aberasturi D, Aizpurua J, et al. Present and future of surface-enhanced Raman scattering. ACS Nano, 2020, 14: 28-117

6 Li D, Yao D, Li C, et al. Nanosol SERS quantitative analytical method: A review. TrAC Trends Anal Chem, 2020, 127: 115885

7 Jin $\mathrm{Y}, \mathrm{Xie} \mathrm{Y}, \mathrm{Wu} \mathrm{K}$, et al. Probing the dynamic interaction between damaged DNA and a cellular responsive protein using a piezoelectric mass biosensor. ACS Appl Mater Interfaces, 2017, 9: 8490-8497

8 Perales-Rondon JV, Colina A, González MC, et al. Roughened silver microtubes for reproducible and quantitative SERS using a templateassisted electrosynthesis approach. Appl Mater Today, 2020, 20: 100710

9 Pilot R. SERS detection of food contaminants by means of portable Raman instruments. J Raman Spectrosc, 2018, 49: 954-981

10 Huang JA, Mousavi MZ, Giovannini G, et al. Multiplexed discrimination of single amino acid residues in polypeptides in a single SERS hot spot. Angew Chem Int Ed, 2020, 59: 11423-11431

11 Kim M, Ko SM, Lee C, et al. Hierarchic interfacial nanocube assembly for sensitive, selective, and quantitative DNA detection with surfaceenhanced Raman scattering. Anal Chem, 2019, 91: 10467-10476

12 Yan W, Yang L, Chen J, et al. In situ two-step photoreduced SERS materials for on-chip single-molecule spectroscopy with high reproducibility. Adv Mater, 2017, 29: 1702893

13 Chen G, Dai Z, Ji B, et al. Dynamic enrichment of plasmonic hot-spots and analytes on superhydrophobic and magnetically functionalized platform for surface-enhanced Raman scattering. Sens Actuat B-Chem, 2020, 319: 128297

$14 \mathrm{Wu}$ Y, Bennett D, Tilley RD, et al. How nanoparticles transform single molecule measurements into quantitative sensors. Adv Mater, 2020, 32: 1904339

15 Li JF, Zhang YJ, Ding SY, et al. Core-shell nanoparticle-enhanced Raman spectroscopy. Chem Rev, 2017, 117: 5002-5069

16 Xu D, Teng F, Wang Z, et al. Droplet-confined electroless deposition of silver nanoparticles on ordered superhydrophobic structures for high uniform SERS measurements. ACS Appl Mater Interfaces, 2017, 9: 21548-21553

17 Kim N, Thomas MR, Bergholt MS, et al. Surface enhanced Raman scattering artificial nose for high dimensionality fingerprinting. Nat Commun, 2020, 11: 207

18 Lee HK, Lee YH, Koh CSL, et al. Designing surface-enhanced Raman scattering (SERS) platforms beyond hotspot engineering: Emerging opportunities in analyte manipulations and hybrid materials. Chem Soc Rev, 2019, 48: 731-756

19 Yang L, Peng Y, Yang Y, et al. A novel ultra-sensitive semiconductor SERS substrate boosted by the coupled resonance effect. Adv Sci, 2019, 6: 1900310

20 Cong S, Yuan Y, Chen Z, et al. Noble metal-comparable SERS en- 
hancement from semiconducting metal oxides by making oxygen vacancies. Nat Commun, 2015, 6: 7800

21 Shan Y, Zheng Z, Liu J, et al. Niobium pentoxide: A promising surfaceenhanced Raman scattering active semiconductor substrate. npj Comput Mater, 2017, 3: 11

22 Guan $\mathrm{H}, \mathrm{Yi} \mathrm{W}, \mathrm{Li} \mathrm{T}$, et al. Low temperature synthesis of plasmonic molybdenum nitride nanosheets for surface enhanced Raman scattering. Nat Commun, 2020, 11: 3889

23 Wang $\mathrm{X}$, Shi W, Wang $\mathrm{S}$, et al. Two-dimensional amorphous $\mathrm{TiO}_{2}$ nanosheets enabling high-efficiency photoinduced charge transfer for excellent SERS activity. J Am Chem Soc, 2019, 141: 5856-5862

24 Ye Y, Yi W, Liu W, et al. Remarkable surface-enhanced Raman scattering of highly crystalline monolayer $\mathrm{Ti}_{3} \mathrm{C}_{2}$ nanosheets. Sci China Mater, 2020, 63: 794-805

25 Yilmaz M, Babur E, Ozdemir M, et al. Nanostructured organic semiconductor films for molecular detection with surface-enhanced Raman spectroscopy. Nat Mater, 2017, 16: 918-924

26 Zheng Z, Cong S, Gong W, et al. Semiconductor SERS enhancement enabled by oxygen incorporation. Nat Commun, 2017, 8: 1993

27 Song G, Gong W, Cong S, et al. Ultrathin two-dimensional nanostructures: Surface defects for morphology-driven enhanced semiconductor SERS. Angew Chem Int Ed, 2021, 60: 5505-5511

28 Huang $\mathrm{C}, \mathrm{Li} \mathrm{A}$, Chen $\mathrm{X}$, et al. Understanding the role of metal-organic frameworks in surface-enhanced Raman scattering application. Small, 2020, 16: 2004802

29 Sun $\mathrm{H}$, Cong S, Zheng Z, et al. Metal-organic frameworks as surface enhanced Raman scattering substrates with high tailorability. J Am Chem Soc, 2019, 141: 870-878

30 Lin J, Yu J, Akakuru OU, et al. Low temperature-boosted high efficiency photo-induced charge transfer for remarkable SERS activity of ZnO nanosheets. Chem Sci, 2020, 11: 9414-9420

31 Lin J, Hao W, Shang Y, et al. Direct experimental observation of facetdependent SERS of $\mathrm{Cu}_{2} \mathrm{O}$ polyhedra. Small, 2018, 14: 1703274

32 Liu H, Yang Z, Meng L, et al. Three-dimensional and time-ordered surface-enhanced Raman scattering hotspot matrix. J Am Chem Soc, 2014, 136: 5332-5341

33 Wang X, Guo L. SERS activity of semiconductors: Crystalline and amorphous nanomaterials. Angew Chem Int Ed, 2020, 59: 4231-4239

34 Sun $\mathrm{J}, \mathrm{Hu} \mathrm{H}$, Zheng $\mathrm{D}$, et al. Light-emitting plexciton: Exploiting plasmon-exciton interaction in the intermediate coupling regime. ACS Nano, 2018, 12: 10393-10402

35 Yang S, Yao J, Quan Y, et al. Monitoring the charge-transfer process in a Nd-doped semiconductor based on photoluminescence and SERS technology. Light Sci Appl, 2020, 9: 117

36 Feng E, Zheng T, He X, et al. A novel ternary heterostructure with dramatic SERS activity for evaluation of PD-L1 expression at the singlecell level. Sci Adv, 2018, 4: eaau3494

37 Liu P, Zhao Y, Qin R, et al. Photochemical route for synthesizing atomically dispersed palladium catalysts. Science, 2016, 352: 797-800

38 Wang J, Li Z, Wu Y, et al. Fabrication of single-atom catalysts with precise structure and high metal loading. Adv Mater, 2018, 30: 1801649

39 Yang J, Li W, Wang D, et al. Electronic metal-support interaction of single-atom catalysts and applications in electrocatalysis. Adv Mater, 2020, 32: 2003300

40 Wang $\mathrm{C}, \mathrm{Li} \mathrm{A}, \mathrm{Li} \mathrm{C}$, et al. Ultrahigh photocatalytic rate at a singlemetal-atom-oxide. Adv Mater, 2019, 31: 1903491

41 Hsu HC, Huang BC, Chin SC, et al. Photodriven dipole reordering: Key to carrier separation in metalorganic halide perovskites. ACS Nano, 2019, 13: 4402-4409

42 Caicedo-Dávila S, Gunder R, Márquez JA, et al. Effects of postdeposition annealing on the luminescence of mixed-phase $\mathrm{CsPb}_{2} \mathrm{Br}_{5} / \mathrm{CsPbBr}_{3}$ thin films. J Phys Chem C, 2020, 124: 19514-19521

43 Su X, Ma H, Wang H, et al. Surface-enhanced Raman scattering on organic-inorganic hybrid perovskites. Chem Commun, 2018, 54: 21342137

44 Hewitt RW, Winograd N. Investigation of the oxidation of polycrystalline lead by XPS and SIMS. Surf Sci, 1978, 78: 1-14

45 Le Ru EC, Etchegoin PG. Single-molecule surface-enhanced Raman spectroscopy. Annu Rev Phys Chem, 2012, 63: 65-87
46 Cook C, Farber-Eger E, Wang T, et al. Prevalence of clinically apparent hypertrophic cardiomyopathy in 32 patients with the gla al43t mutation: Implications for genetic screening for fabry disease in patients with hypertrophic cardiomyopathy. J Am College Cardiol, 2015, 65: A953

47 Kim JY, Lee JW, Jung HS, et al. High-efficiency perovskite solar cells. Chem Rev, 2020, 120: 7867-7918

48 Li Y, Xu M, Xia Y, et al. Multilayer assembly of electrospun/electrosprayed PVDF-based nanofibers and beads with enhanced piezoelectricity and high sensitivity. Chem Eng J, 2020, 388: 124205

49 Osticioli I, Mencaglia AA, Siano S. Temperature-controlled portable Raman spectroscopy of photothermally sensitive pigments. Sens Actuat B-Chem, 2017, 238: 772-778

$50 \mathrm{Yu} \mathrm{Z,} \mathrm{Yu} \mathrm{W,} \mathrm{Xing} \mathrm{J,} \mathrm{et} \mathrm{al.} \mathrm{Charge} \mathrm{transfer} \mathrm{effects} \mathrm{on} \mathrm{resonance-en-}$ hanced Raman scattering for molecules adsorbed on single-crystalline perovskite. ACS Photonics, 2018, 5: 1619-1627

51 Cañamares MV, Lombardi JR. Raman, SERS, and DFT of mauve dye Adsorption on Ag nanoparticles. J Phys Chem C, 2015, 119: 1429714303

52 Granold M, Hajieva P, Toșa MI, et al. Modern diversification of the amino acid repertoire driven by oxygen. Proc Natl Acad Sci USA, 2018, 115: 41-46

53 Yang L, Jiang X, Ruan W, et al. Observation of enhanced Raman scattering for molecules adsorbed on $\mathrm{TiO}_{2}$ nanoparticles: Chargetransfer contribution. J Phys Chem C, 2008, 112: 20095-20098

54 Saunders SR, Eden MR, Roberts CB. Modeling the precipitation of polydisperse nanoparticles using a total interaction energy model. J Phys Chem C, 2011, 115: 4603-4610

55 Demirel G, Gieseking RLM, Ozdemir R, et al. Molecular engineering of organic semiconductors enables noble metal-comparable SERS enhancement and sensitivity. Nat Commun, 2019, 10: 5502

Acknowledgements This work was supported by the Natural Science Foundation of Beijing Municipality (Z180014). We thank Dr. Chen Qian and Dr. Yingying Wang for providing measurement support, and we also thank Taifeng Lin, Prof. Yiyang Sun and Prof. Shengbai Zhang for discussions.

Author contributions Feng $\mathrm{R}$ designed and performed the experiments; Miao Q analyzed TAS data; Zhang X calculated the electronic structure; Cui $\mathrm{P}$ analyzed the XANES data; Wang $\mathrm{C}$ wrote the paper; Han $\mathrm{X}$ discussed partial experimental data. All authors contributed to the general discussion.

Conflict of interest The authors declare that they have no conflict of interest.

Supplementary information Experimental details and supporting data are available in the online version of the paper.

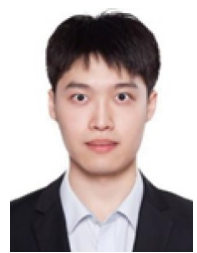

Ran Feng received his BSc degree in 2020 from Nanjing Normal University, China. He is currently an MSc candidate in physics under the supervision of Prof. Cong Wang at Beijing Key Laboratory of Microstructure and Properties of Solids, Beijing University of Technology. His research centers on developing the single-site decorated SERS substrate for ultrasensitive molecule detection.

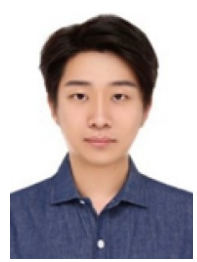

Qing Miao received his BSc degree in engineering from Baicheng Normal University in 2014 and MSc degree in physics from North China Electric Power University in 2017. He is currently pursuing his $\mathrm{PhD}$ degree in optical engineering under the supervision of Prof. Dawei He at Beijing Jiaotong University. His research focuses on ultrafast laser spectroscopy of nanoscale materials, such as photocarrier dynamics, charge transfer, and its potential applications in optoelectronics and photonics. 


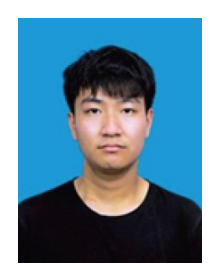

Xiang Zhang received his BSc degree in 2020 from Sichuan University of Science \& Engineering. Then, he joined the Institute of Structure \& Function, Chongqing University as a master student. His research centers on Schottky barrier heights in two-dimensional field-effect transistors.

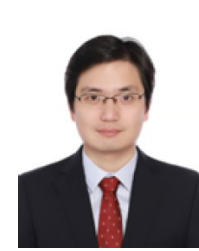

Peixin Cui received his $\mathrm{PhD}$ degree from the University of Science and Technology of China in 2013, followed by working as a postdoc at the same university. He joined the Institute of Soil Science, Chinese Academy of Sciences in 2016. His research focuses on the study of local structures of nano and single-atom materials by synchrotron radiation techniques.

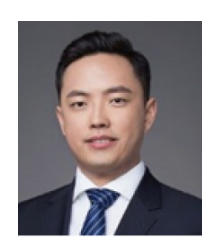

Cong Wang received his BSc degree in 2009 and MSc degree in 2012 from the University of Science and Technology Beijing, respectively. In 2015, he received his $\mathrm{PhD}$ degree from Hong Kong University of Science and Technology. Then, he joined Beijing University of Technology and became an associate professor in 2016. His research interests focus on the single-atom sites rational design and synthesis, single-sites catalysis, and single-sites SERS in energy, environment and biology.

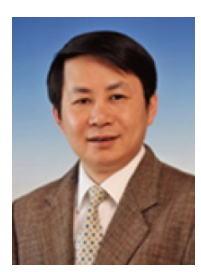

Xiaodong Han received his BSc degree in 1989 and MSc degree in 1992 from Harbin Institute of Technology. In 1996, he received his $\mathrm{PhD}$ degree from Dalian University of Technology. Then, he joined City University of Hong Kong and the University of Pittsburgh as postdoc. In 2001, he joined HKL Technology (Oxford Instruments Group), and in 2004, he joined Beijing University of Technology. His research interests focus on in-situ transmission electron microscopy, and physical and chemical properties of materials in catalytic, optical, and mechanical communities.

\section{钲钛矿上单原子位点实现高灵敏定量化表面拉曼增强}

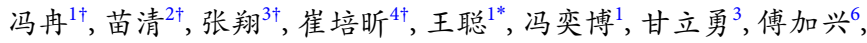
王仕博7, 戴子忆 ${ }^{8}$, 胡利明 5 , 罗云敬 ${ }^{5}$, 孙伟海 ${ }^{7}$, 张小姻 ${ }^{2}$, 肖家文 ${ }^{1}$, 巫金波 ${ }^{6}$, 周冰朴 ${ }^{8}$, 邹明强 ${ }^{9}$, 何大伟 $^{2}$, 周小元 $^{3}$, 韩晓东 ${ }^{1^{*}}$

摘要 表面增强拉曼散射(SERS)是一种快速且无损的化学和生物分子 检测技术. 高灵敏且定量化的SERS检测技术对其在实际中的应用至关 重要, 尤其是对生物分子如氨基酸和碱基的便携化检测. 但是鲜有技术 手段能够实现对这些最基本的生物分子进行高灵敏且定量化的拉曼检 测. 我们提出了一种无需贵金属的单原子位点修饰芯片策略, 用铇单原 子氧化物修饰铅卤钙铁矿芯片, 实现了多种检测物的高灵敏定量化识 别, 包括罗丹明、酪氨酸和胞嘧啶. 芯片上单原子位点技术能够实现对 罗丹明、酪氨酸和胞嘧啶的线性检测, 相应的定量化区间分别为: $10^{-6}-1 \mathrm{mmol} \mathrm{L}^{-1}, 0.06-1 \mathrm{mmol} \mathrm{L}^{-1}$ 和0.2-45 $\mathrm{mmol} \mathrm{L}^{-1}$, 同时我们的拉曼 增强芯片对这三种分子的增强因子在非等离子激元半导体中是最高 的. 我们通过实验测试结合理论模拟揭示了由可控单原子位点实现拉 曼增强的机理: 束缚由钙钛矿基底产生的光生电子, 同时高效且定量地 将这些电子转移到待测分子上. 不仅如此, 芯片上单原子位点技术可以 在便携式拉曼设备下得到与台式拉曼设备类似的增强效果，这意味着 这项技术有机会应用于多种生物分子的低成本、广谱、即时检测和体 外检测. 